\title{
La délicate application de la volonté populaire
}

\section{Bertrand Graz}

Médecin agréé, Centre hospitalier universitaire vaudois (CHUV) et Faculté de médecine et biologie (FBM), Lausanne

Le peuple a voté et l'a fait inscrire en 2009 dans la Constitution suisse (article 118a): il faut «prendre en compte» (sic) les médecines complémentaires (MC). Oui, mais comment et dans quel délai? L'heure est au débat car une nouvelle mouture de l'ordonnance d'application de la Loi sur l'assurance maladie (LaMal) est en consultation [1], qui admet cinq MC dans l'assurance de base bien que les évaluations demandées n'aient pas abouti. Après avoir participé aux efforts pour mettre sur pied au Centre hospitalier universitaire vaudois (CHUV) une concrétisation du vote populaire de 2009 sur les MC, je tente ici un bilan de l'expérience lausannoise et quelques réflexions afin d'informer le débat. Ce qui est en jeu c'est la tâche délicate d'appliquer dans notre système de santé une volonté exprimée démocratiquement et traduite par un article de la Constitution.

D'abord le bilan. Sept ans après la votation, ou en sommes-nous avec les MC au CHUV et à l'Ecole de médecine de Lausanne? Qu'on en juge sur trois questions:

1. Propose-t-on aux patients, quand c'est adéquat, celles des MC qui, à la suite de bonnes études cliniques, apparaissent clairement sûres, efficaces et économiques?

Non, on n'a pas réussi à établir cette équation de manière systématique dans la pratique. L'offre de MC dépend d'initiatives isolées dans les Services, en

\section{Die schwierige Anwendung des Volkswillens}

Eine neue Version der Ausführungsbestimmungen des KVG, die die Aufnahme von fünf komplementärmedizinischen Disziplinen (KM) in die Grundversicherung betrifft, ist in eidgenössischer Vernehmlassung. Die Auswertung dieser Disziplinen wurde aber nicht voll ausgeführt und es wurde beschlossen, diese KM als Ganzes zu akzeptieren aufgrund des "Vertrauensprinzips», das auch für konventionelle Medizin angewendet wird. Dies bedeutet, dass Leistungen in das öffentliche Gesundheitssystem integriert werden, die gemäss unseren klassischen randomisierten Studien die gleiche Wirksamkeit wie Plazebo haben. Die schwierige Anwendung des Volkswillens im Bereich der KM lädt uns ein, über dieses scheinbare Paradox nachzudenken: das "wirksame und kostengünstige Plazebo». général liées à la présence d'une personne et disparaissant avec son départ. Il est pourtant éthiquement défendable de dire que le médecin devrait proposer à son patient tout traitement potentiellement utile et pas trop cher, qu'il soit conventionnel ou complémentaire. Le facteur limitant semble être fréquemment que les responsables de Services n'ont pas connaissance des études démontrant que des MC dans leur domaine sont désormais «evidence-based».

La volonté populaire de 2009 a-t-elle été respectée et mise en application?

2. Les $M C$ douteuses, à efficacité possible mais pas établie, sont-elles évaluées scientifiquement?

Non, on n'a pas pu développer cette ligne de recherche; elle est remise à plus tard dans l'institution lausannoise, à cause du coût de telles études. A la place d'évaluations cliniques on a privilégié des enquêtes sur les usages de MC et sur les opinions à leur sujet.

3. A quoi sert la modeste somme dépensée (un tiers de million de francs par an) pour le «Centre des médecines intégrative et complémentaire» responsable de la question des MC au CHUV / Faculté de médecine?

Sous le vocable "coordination clinique» sont rassemblées des personnes intéressées aux MC, dont la marge de manœuvre reste limitée par le système qui veut que ce sont d'abord les Services de chaque spécialité médicale qui décident des soins offerts. Outre les enquêtes citées plus haut (parfois sous forme de travail de master ou de thèse), nous avons instauré à Lausanne, comme à Genève, un enseignement informatif sur quelques MC (les cours obligatoires représentent huit heures sur l'ensemble du cursus; une trentaines d'étudiants peuvent aussi suivre un cours à option).

Le bilan reste donc assez limité. La volonté populaire de 2009 a-t-elle été respectée et mise en application? L'auteur de ces lignes émettait il y a sept ans l'hypo- 
thèse qu'on était prêt à "profiter pleinement de l'expansion rapide des connaissances disponibles sur les MC, sur leurs potentiels et sur leurs limites, de manière à travailler de la façon la plus bénéfique possible pour nos patients» [2]. Hypothèse démentie par les faits. Pendant que les Facultés de médecine, priées par voie de motion parlementaire, ajustaient leur enseignement, à l'hôpital j'ai plutôt eu le sentiment d'assez fréquentes résistances à l'implantation de MC dans la pratique, même quand il s'agissait de prestations dûment validées par la recherche clinique. L'argument le plus souvent entendu était que les MC seraient «sans aucune preuve scientifique» - alors que cette affirmation doit aujourd'hui être nuancée, au vu des recherches publiées.

On voit ici un exemple d'un problème plus général en Suisse: la difficulté à faire appliquer les décisions démocratiques. Qu'on pense au quota de résidences secondaires dans les communes touristiques ou à l'accès aux rives des lacs pour le public, c'est toujours pareil: une forte minorité résiste pied à pied alors que le peuple souverain a clairement exprimé son choix devenu législation. Dans le cas des MC, il s'agit surtout de praticiens académiques, que j'ai eu l'occasion d'entendre à de nombreuses reprises dans les hôpitaux suisses professer avec beaucoup d'assurance que les MC sont «toutes des placebos» et user de leur influence parmi les cadres hospitaliers pour empêcher que des MC soient pressenties pour le catalogue des prestations officielles.

Si on regarde d'un peu plus près, la difficulté à établir une utilisation cohérente des MC dans le cadre de la médecine académique reflète deux contradictions inhérentes à notre système de santé et à la médecine. En premier, la contradiction entre la volonté affichée de choisir nos soins sur des bases scientifico-économiques et la réalité historique du principe de confiance qui confie au médecin le libre choix des prestations qu'il offre. En deuxième, la contradiction inhérente à la notion d'«efficacité du placebo», développée plus loin. Première contradiction, celle de la science et de la confiance: on se souvient qu'il avait été demandé au début des années 2000, sous la forme d'un programme de recherche national, d'évaluer scientifiquement les MC pour savoir lesquelles devaient être remboursées par l'assurance maladie de base. Suivant l'avis de la Commission fédérale des prestations, le Département fédéral de l'intérieur avait suspendu le remboursement des MC en 2005. Certains praticiens MC prétendaient que leur discipline était impossible à évaluer par des études cliniques comparatives. D'autres étaient favorables à une évaluation académique classique, mais notaient que cela prendrait énormément de temps. Bref: la voie à laquelle on aboutit actuellement est celle qui sauvegarde l'autonomie du médecin pratiquant une $\mathrm{MC}$ et l'autorise à traiter ses patients comme il l'entend, à condition qu'il ait suivi une formation adéquate (actuellement attestation FMH de formation complémentaire - AFC). Le principe de confiance («Vertrauensprinzip») justifie l'acceptation de disciplines entières de MC dans le catalogue des soins remboursés par l'assurance de base. Seules les prestations controversées doivent être contrôlées. C'est un renversement du fardeau de la preuve. Ainsi, comme on fait confiance aux Chefs de Service des hôpitaux pour décider quels soins doivent être offerts dans leur spécialité, de même on fait confiance aux médecins spécialistes de MC pour les cinq disciplines qui ont été admises globalement: médecine anthroposophique, acupuncture, médecine traditionnelle chinoise, homéopathie et phytothérapie.

\section{La question de l'«efficacité du placebo» est un point essentiel dans la réflexion sur les MC.}

Si le principe de confiance satisfait les défenseurs des MC [3], il faut reconnaître aussi que «de nombreuses procédures de la médecine allopathique ne répondent pas, ou plus, aux trois critères» de la LaMal (article $32 \rightarrow$ efficacité, adéquation, économicité) et «l'évaluation du bénéfice réel pour le patient est souvent inexistante» [4]. La LaMal devrait-elle être comprise comme un idéal que nous n'atteindrons jamais? On se rappelle que la plupart des études cliniques sur l'homéopathie montrent que son efficacité est au niveau du placebo. Or, cela ne signifie pas efficacité négligeable. La question de l'«efficacité du placebo» est un point essentiel dans la réflexion sur les MC.

Puisqu'il a été beaucoup utilisé en recherche médicale comme référence de l'absence d'efficacité spécifique, on a tendance à l'oublier: le placebo peut avoir une efficacité. Ceci a été montré même dans un cas où le patient n'était pas trompé et savait qu'il recevait un placebo [5]. On connaît un peu mieux désormais les mécanismes d'action du placebo, notamment au niveau de l'interface psycho-somatique par où il peut agir finalement sur les mêmes récepteurs que les traitements conventionnels [6]. Une partie des traitements MC admis officiellement sont certes des placebos; ils pourraient cependant s'avérer efficaces, appropriés et économiques et ainsi remplir les critères de la LaMal. Vue sous cet angle, une "étude contre placebo" classique ne suffit plus à se déterminer pour valider un traitement. On devrait plutôt comparer les approches thérapeutiques entre elles, leurs effets primaires et secondaires, leurs coûts, leur facilité d'usage, etc. pour 
produire les informations utiles au dialogue avec le patient.

Ainsi la deuxième contradiction, historique on l'a vu, est celle de l'efficacité du placebo. Peut-être que l'ancien médecin cantonal vaudois ne dit pas autre chose quand il déclare «Je n'ai pas de lien avec l'homéopathie mais j'ai entendu trop de témoignages crédibles pour décréter qu'elle ne sert à rien du tout» [7]. Pour avancer, il faudrait étudier les rapports coût-efficacité ou coûtutilité de placebos proposés en remplacement ou en complément de traitements conventionnels, par exemple pour le bien-être ou accélérer une guérison attendue.

\section{Il s'agit donc de nous enlever de la tête le jugement péjoratif attaché au placebo et de voir quand il peut être utile.}

L'acceptation en Suisse de cinq disciplines complètes de MC dans l'assurance de base signifie qu'on valide du même coup parmi elles l'usage de certains traitements qui, selon nos études cliniques classiques, apparaissent comme de purs placebos. Ce qui ne les empêche pas d'avoir une action physiologique réelle, d'apporter éventuellement un bénéfice au patient et de présenter dans certains cas un rapport coût/efficacité intéressant. Il s'agit donc de nous enlever de la tête le jugement péjoratif attaché au placebo et de voir quand il peut être utile. J'ai même entendu des médecins déclarer que le placebo est le meilleur remède: quand il est suffisant, il est le plus sûr des traitements. Ce sera une petite révolution dans notre conception des soins: travailler avec des prestataires de MC et même des traitements qui, du point de vue des études cliniques, seraient des placebos, mais des placebos coût-efficaces. Une contradiction dans les termes? Plutôt une nouvelle façon de penser, qui fait son chemin en physiologie, en neuro-psychologie et, plus lentement, en médecine.

En conclusion, dans l'exemple présenté ici de l'hôpital universitaire à Lausanne, l'inscription il y a sept ans dans la Constitution d'un article demandant la prise en compte des médecines complémentaires a été suivie de peu de changement dans la pratique clinique. L'actuelle consultation sur la nouvelle ordonnance d'application de la Loi sur l'assurance maladie nous invite à réfléchir au rôle de traitements non-conventionnels dans notre système de santé, y compris ceux qui pourraient apparaître à la fois comme "coût-efficaces» et "placebos».

\section{Références}

1 «Le Département fédéral de l'intérieur (DFI) souhaite mettre les médecines complémentaires au même niveau que les autres disciplines médicales. Il lance aujourd'hui la procédure d'audition concernant les modifications d'ordonnances correspondantes.» Cf: http://www.admin.ch/ch/f/gg/pc/pendent.html (accédé le 12 avril 2016)

2 Graz B, Schopper D: Médecines complémentaires: vers un consensus "evidence-based" à l'hôpital universitaire. Revue Médicale Suisse No. 229, 9 décembre 2009.

3 Fédération de la médecine complémentaire (Fedmedcom - en allemand Dachverband Komplementärmedizin - Dakomed): http://www.fedmedcom.ch/

4 Genton B, Loutan L: Efficacité, adéquation, économicité: la leçon des pays pauvres aux nantis... Revue Médicale Suisse No. 294, 11 mai 2011: 981-2.

5 Petkovic G, Charlesworth JEG, Kelley J, Miller F, Roberts N, Howick J. Effects of placebos without deception compared with no treatment: protocol for a systematic review and meta-analysis. BMJ Open. 2015;5(11):e009428.

6 Benedetti F. Placebo and the New Physiology of the Doctor-Patient Relationship. Physiological Reviews. 2013;93(3):1207-46.

7 Martin J. Les médecines complémentaires dans les hôpitaux universitaires. Bull Méd Suisses 2016;97(10):390. 